Pteridines

Vol. 15, 2004, pp. $113-119$

\title{
Elevated Post-transplant Levels of Neopterin are Associated with Poorer long-term Graft Outcome*
}

\author{
S. O. Grebe', U. Kuhlmann', T. F. Müller ${ }^{1,2}$ \\ Clinic of Internal Medicine, Department of Nephrology, Philipps-University, Marburg, Germany \\ 'Department of Medicine, Division of Nephrology, Brigham and Women's Hospital, Boston, MA, USA
}

* Dedicated to Prof. Dr. Dr. h. c. Helmut Wachter on the occasion of his $75^{\text {th }}$ birthday

\begin{abstract}
Chronic allograft nephropathy (CAN) and death with functioning graft due to cardiovascular disease (CVD) are the key determinants for long-term renal allograft survival. Chronic allograft nephropathy and cardiovascular disease are seen as manifestations of a single disease entity with a common pathogenesis including inflammation and accelerated atherogenesis. Therefore we investigated the relation between early post-transplant inflammatory burden and long-term graft survival.

In 64 consecutive renal transplant patients the acute phase reactants serum amyloid A (SAA) and serum C-reactive protein (S-CRP) as well as the macrophage product neopterin in urine (U-NEOP) and serum (S-NEOP) were determined daily during the immediate postoperative period (mean p.o.obs. $x=29.2 \pm 8.7$ days, total of $\Sigma=1869$ days). SAA and CRP were measured with high-sensitive assays (in $\mathrm{mg} / \mathrm{l}$; immune nephelometry, Dade Behring Co., Marburg, Germany), NEOP was measured with ELISA-technique (Brahms, Berlin, Germany) and related to serum and urine creatinine levels, resp. (in $\mu \mathrm{mol} / \mathrm{mol}$ creatinine). The association between the mean values of these parameters and the survival distribution function of the 64 patients was tested using the log rank test and the Wilcoxon-test. In this analysis graft loss was defined as either resumption of dialysis treatment or patient death with functioning graft.

The 1- and 5- year graft survival rates in our patients were $93 \%$ and $76 \%$, resp. The markers showed the following mean post-transplant levels: S-CRP $x=21.3 \pm 16.1 \mathrm{mg} / \mathrm{l}, \mathrm{SAA} x=10.1 \pm 6.5 \mathrm{mg} / \mathrm{l}, \mathrm{U}-\mathrm{NEOP} \mathrm{x}=602 \pm 427$ $\mathrm{mmol} / \mathrm{mol}$ creatinine and S-NEOP $\mathrm{x}=81 \pm 60 \mu \mathrm{mol} / \mathrm{mol}$ creatinine. Both log rank test and Wilcoxon-test provided evidence that the graft survival is negatively related to the post-transplant levels of U-NEOP $(p=0.009$ and $p=0.027$, resp.). The markers S-NEOP $(p=0.074$ and $p=0.116$, resp.), SAA $(p=0.599$ and $p=0.294$, resp.), and S-CRP ( $p=0.059$ and $p=0.358$, resp.) did not reach statistical significance.

These findings support the impact of the inflammatory burden on graft survival. In particular, elevated post-transplant neopterin values, reflecting activated innate and adaptive responses, are predictive for long-term graft outcome.
\end{abstract}

Key words: neopterin, transplantation, graft survival, nephropathy

\section{Introduction}

Long-term survival of kidney allografts has not improved substantially over the last decade despite numerous new immunosuppressive strategies (Häyry 1999. Hariharan 2000). The most frequent cause of renal allograft loss beyond the early post-transplant period is either chronic allograft nephropathy (CAN) or death with functioning graft due to cardiovascular disease (CVD) (Hariharan 2000. Kreis 2001).

Chronic allograft nephropathy and cardiovascuar disease can be seen as manifestations of a single disease entity with a common pathogenesis including inflammation and accelerated atherogenesis (Häyry 1999, Furness 2001, Morris 2001, Avery 2003).

The marked production of various inflammatory

Correspondence to: PD Dr. Thomas F. Mueller, Brigham and Women's Hospital, PBB-170, Laboratory of Molecular Immunology, 75 Francis Strcet, Boston, MA 02115, Phone: ++1 6177325252 , Fax: +1 6172646870 , e-mail: tmueller@rics.bwh.harvard.edu 
markers and cytokines in response to inflammation and injury has been well described in atherosclerotic vascular disease (Ross 1999). The association of the acute phase reactants C-reactive protein (CRP) and serum amyloid A (SAA) as well as the macrophage product neopterin (NEOP) with different clinical manifestations of atherosclerosis have been examined in great detail (Weiss 1994, Erren 1999, Auer 2001, Ridker 2001, Murr 2002, Delanghe 2002). These findings seem to apply to the field of organ transplantation, too. It has been shown that CRP is of predictive value for the development of transplant coronary artery disease and graft survival in heart transplantation (Eisenberg 2000, Labarrere 2002). Similar results of the predictive ability of CRP could be demonstrated in renal transplantation (Perez 2000, Sezer 2002, Fink for graft and patient outcome in addition to established immunologic and non-immunologic risk factors (Golbabaie 2001, Fellström 2001, Paul 2001). Therefore we investigated the relation between early post-transplant inflammatory burden and long-term graft and patient survival.

\section{Methods}

The presented study is a retrospectivc analysis of 64 consecutive renal allograft recipients. All patients were transplanted and followed up at the University Hospital of the Philipps-University Marburg, Germany. The basic patient characteristics are summarized in (Tab. 1).

Table 1. Patient characteristics, the basic characteristics of the investigated study population are summarized. All parameters are given as mean values + standard deviation.

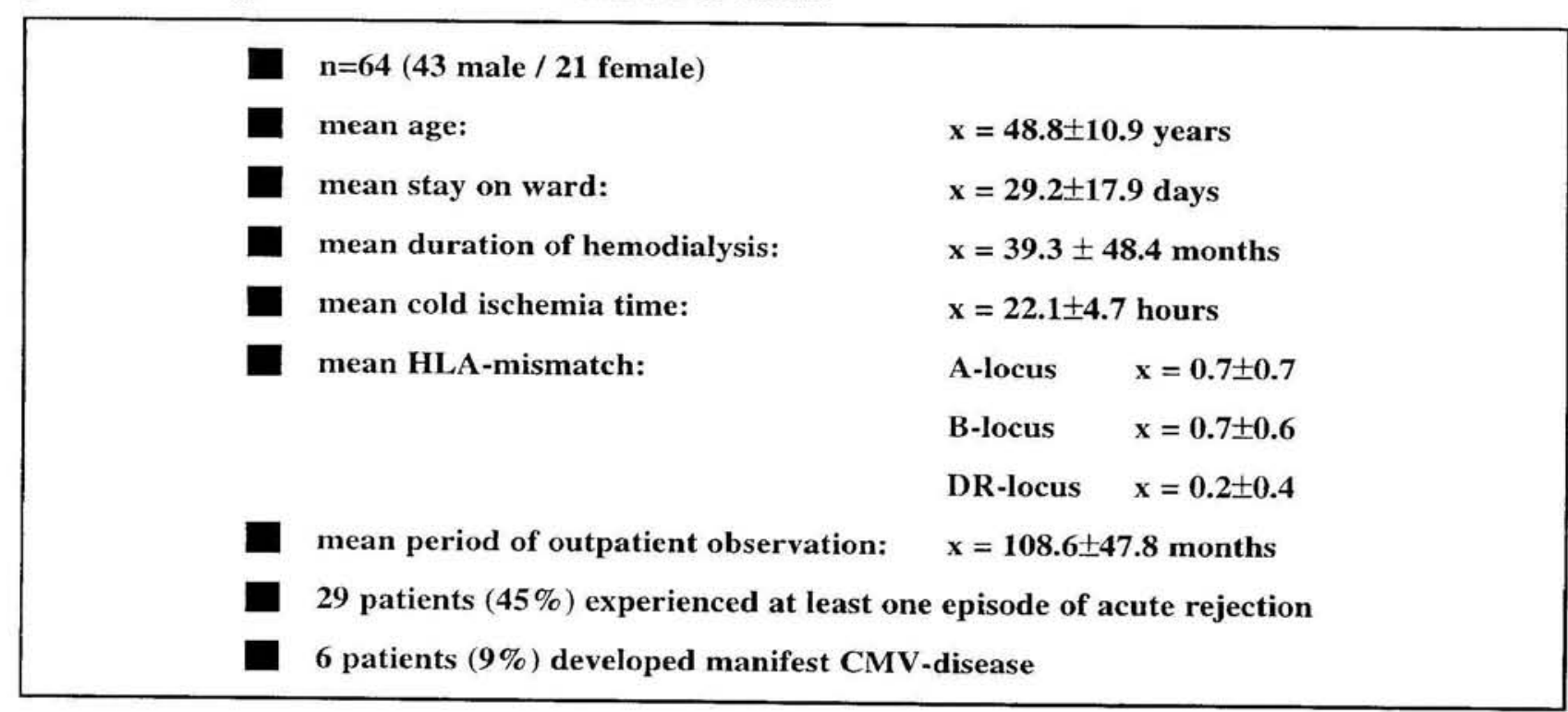

2002, Teppo 2003).

Only few data exist on the prognostic significance of other markers of inflammation. SAA and NEOP appear to be of greater importance in the differential diagnosis of acute complications after kidney transplantation (Grebe 2002, Neumann 2002).

However, one previous report showed that NEOP is a reliable marker not only for the detection of acute rejection episodes but also for long-term graft survival after renal transplantation (Reibnegger 1991). In addition, more recent studies stressed the implication of macrophage activation for short-term renal allograft function and graft survival (Srinivas 2004).

Hence, evidence is emerging that inflammatory burden and accelerated atherosclerosis play a major role
In these patients various clinical and laboratory parameters were measured daily in the immediate postoperative course after transplantation. The inflammatory burden was assessed by calculating the mean serum and/or urine level from all measurements performed throughout the stay on ward. The acute phase proteins SAA and CRP were determined using high sensitive nephelometric assays (in $\mathrm{mg} / \mathrm{l}$; immune nephelometry, Dade Behring Co., Marburg, FRG). The macrophage product NEOP was measured in 24-hour urine (U-NEOP) and serum (S-NEOP) samples by ELISA-technique (in $\mu / \mathrm{mol}$ creatinine; ELISA, Brahms, Berlin, FRG).

For detection of infections with cytomegalovirus (CMV) direct and indirect tests (ELISA, PCR, and Westernblot) were performed at least twice weekly 
during the patients' stay on ward. Unless biopsyproven acute rejection episodes (ASR) were defined as steroid sensitive rises in serum creatinine without other explanation. In addition, independent risk factors for chronic allograft nephropathy like age, sex, HLA-mismatches (HLA-MM), CMV-serostatus, cold ischemia period (CIP), delayed graft function (DGF), and duration of hemodialysis treatment (HD) were recorded.

After discharge all patients were seen at regular intervals in our outpatient clinic. Graft function and cardiovascular complications were documented in addition to the other routine clinical monitoring. The study end point graft loss was defined as either return to chronic dialysis treatment due to chronic allograft nephropathy (graft survival) or patient death with functioning graft due to cardiovascular disease (patient survival).

For all parameters descriptive statistics were calculated. On the basis of the parameter distribution certain cut-off values were established using the upper quartile to divide the study population into two different groups. The mean values of these subgroups were compared using nonparametric tests. For survival analysis the Kaplan-Meier-method was computed. The association between the examined parameters and the outcome rates as well as the comparison of the survival curves of the different subgroups were performed by two different rank tests (log rank test [generalized Savage-test] and Wilcoxon-test). The complete statistical analysis was computed by the statistical software package SAS (Version 8.2, The SAS Institute, Cary,
NC, USA).

The underlying work was carried out in accordance with the standards of the local ethical committee and with the Helsinki Declaration of 1975, as revised in 1983. All patients gave their informed consent.

\section{Results}

The survival curves are shown in (Fig. 1). The 1- and 5- year graft survival rates in our patients were $93 \%$ and $76 \%$, resp. The patient survival rates were $96 \%$ and $81 \%$, resp. (Fig. 1).

The investigated markers showed the following mean post-transplant levels: S-CRP $\mathrm{x}=21.3 \pm 16.1$ $\mathrm{mg} / \mathrm{l}, \mathrm{SAA} \mathrm{x}=10.1 \pm 6.5 \mathrm{mg} / \mathrm{l}$, U-NEOP $\mathrm{x}=602 \pm 427$ $\mu \mathrm{mol} / \mathrm{mol}$ creatinine and S-NEOP $\mathrm{x}=81 \pm 60$ $\mu \mathrm{mol} / \mathrm{mol}$ creatinine.

Both the log rank test and the Wilcoxon-test provided evidence that the graft survival is negatively related to the post-transplant levels of U-NEOP ( $p=0.009$ and $p=0.027$, resp.). The markers S-NEOP $(p=0.074$ and $p=0.116$, resp.), SAA ( $p=0.599$ and $p=0.294$, resp.), and S-CRP ( $p=0.059$ and $p=0.358$, resp.) did not reach statistical significance. Similar findings were seen in the patient survival. Here the calculated p-values were: U-NEOP ( $p=0.01$ and $p=0.009$, resp. $)$, SNEOP ( $p=0.082$ and $p=0.145$, resp.), SAA $(p=$ 0.699 and $p=0.548$, resp.), and S-CRP $(p=0.061$ and $\mathrm{p}=0.558$, resp.) (Tab. 2).

The upper quartiles of the analysed parameters (U-

Table 2. Mean parameter values and association with survival rates, the mean parameter values \pm standard deviation are given. The p-values of the log rank and Wilcoxon-test for the association of the different markers with graft and patient survival are listed. P-values of less than 0.05 are considered to be statistically significant.

\begin{tabular}{|c|c|c|c|}
\hline Parameter & Mean value \pm STD & $\begin{array}{c}\text { Graft survival (p-value) } \\
\text { (Wilcoxon-/log rank test) }\end{array}$ & $\begin{array}{l}\text { Patient survival (p-value) } \\
\text { (Wilcoxon-/log rank test) }\end{array}$ \\
\hline SAA [mg/l] & $10.1 \pm 6.5$ & $0.294 / 0.599$ & $0.548 / 0.699$ \\
\hline CRP [mg/] & $21.3 \pm 16.1$ & $0.358 / 0.059$ & $0.558 / 0.061$ \\
\hline $\begin{array}{l}\text { S-NEOP } \\
\text { [mmol/mol } \\
\text { creatinine] }\end{array}$ & $81 \pm 60$ & $0.116 / 0.074$ & $0.145 / 0.082$ \\
\hline $\begin{array}{l}\text { U-NEOP } \\
\text { [mmol/mol } \\
\text { creatinine] }\end{array}$ & $602 \pm 427$ & $0.027 / 0.009$ & $0.022 / 0.009$ \\
\hline
\end{tabular}



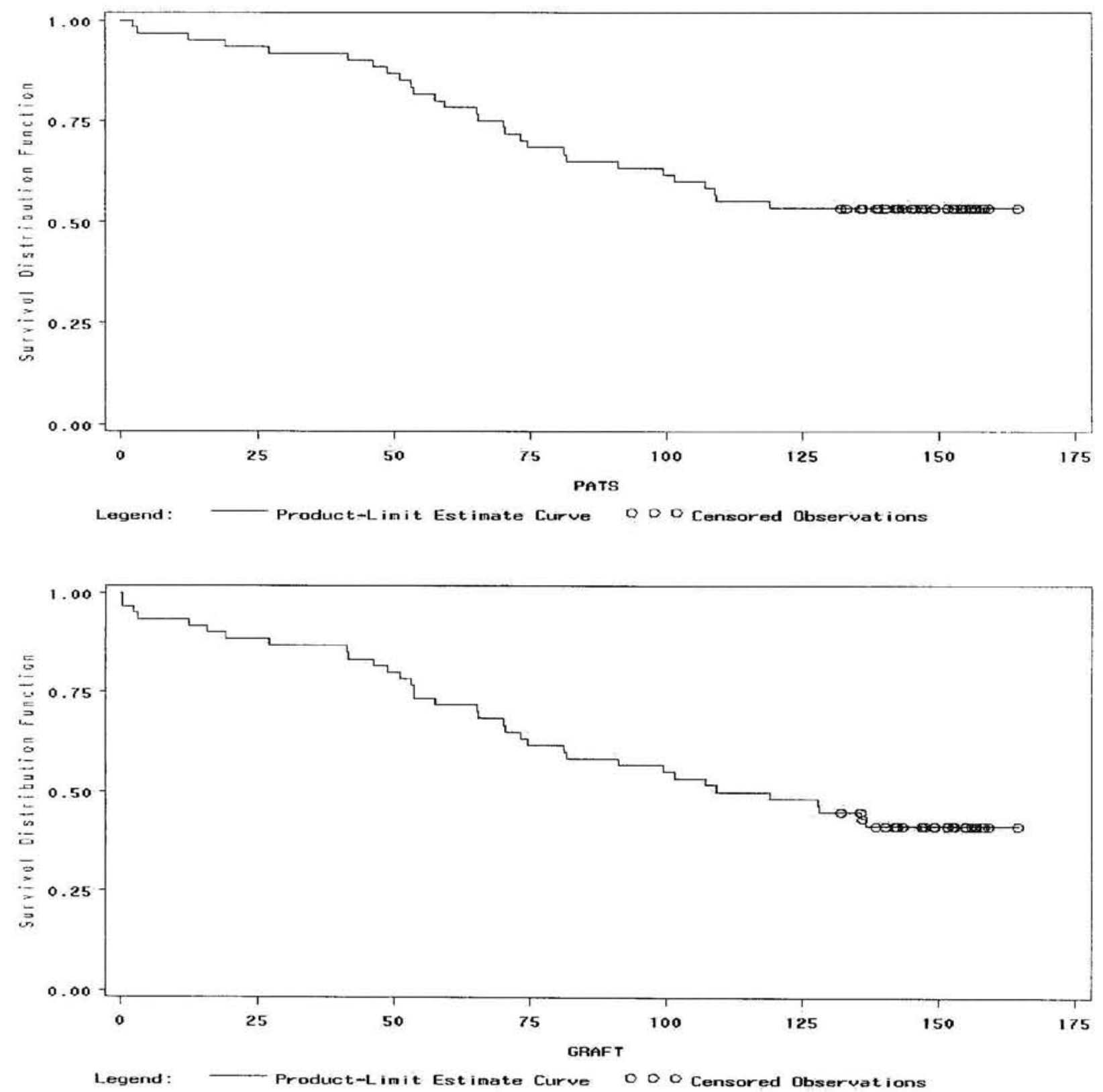

Figure 1. Patient and graft survival, the survival curves for patient (upper) and graft (lower) survival are plotted versus time (months).

NEOP of $800 \mu \mathrm{mol} / \mathrm{mol}$ creatinine, S-NEOP of 106 $\mu \mathrm{mol} / \mathrm{mol}$ creatinine, SAA of $13 \mathrm{mg} / \mathrm{l}$, and CRP of 28 $\mathrm{mg} / \mathrm{l}$ ) were used as cut-off values to divide the study population into different subgroups. The survival curves were compared for these groups. In accordance to the previous results only the upper quartile of $U$ NEOP values discriminated significant differences regarding graft $(\mathrm{p}=0.029)$ and patient survival $(p=0.042)$ (Fig. 2 and 3 ).

\section{Discussion}

Limitations in the long-term graft survival due to chronic allograft nephropathy (CAN) and cardiovascular disease (CVD) are still a major problem of organ transplantation. Numerous new immunosuppressive drugs and treatment strategies have not changed the 

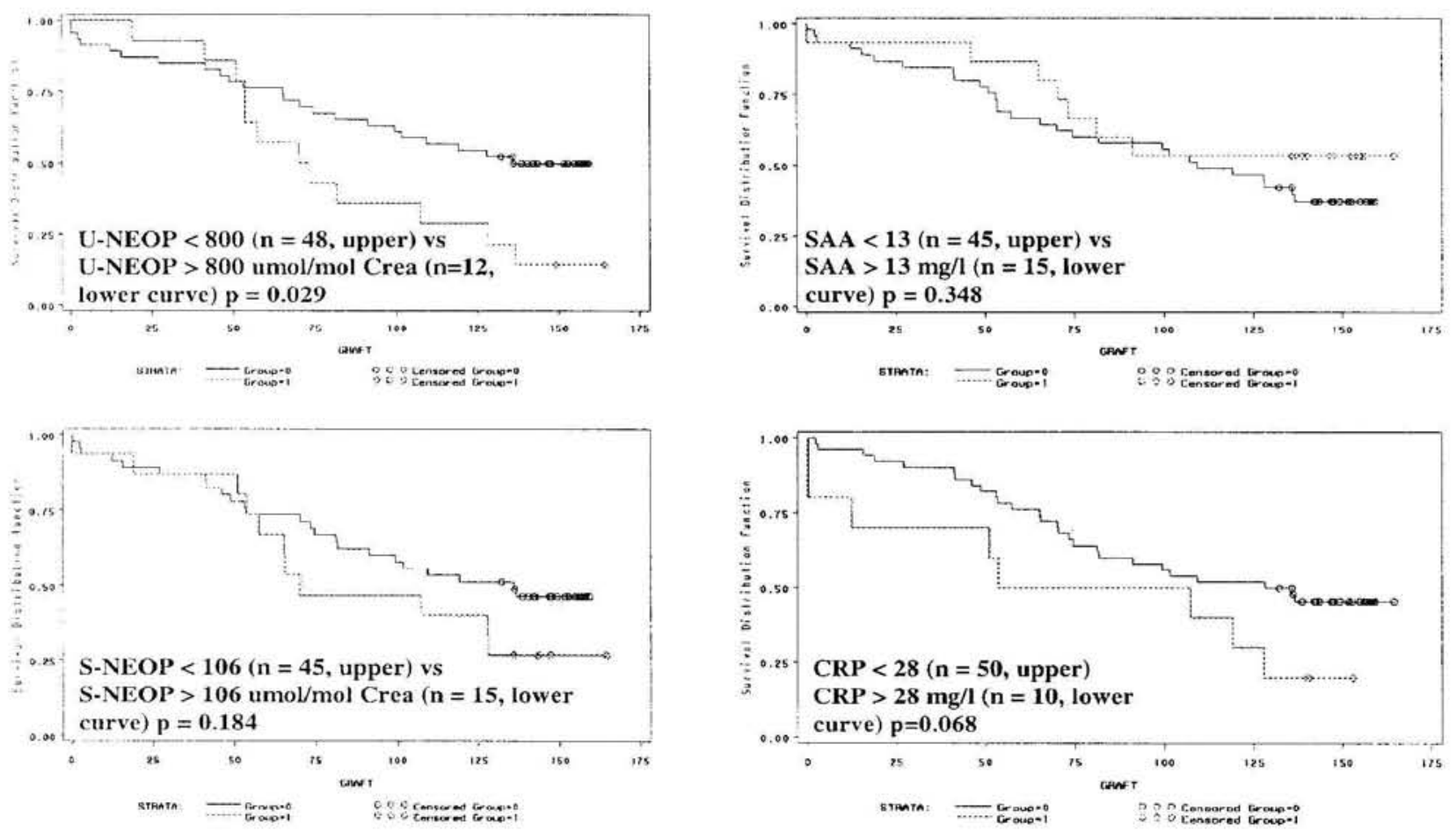

Figure 2. Graft survival depending on mean post-transplant parameter values, the graft survival curves of the different subgroups of patients separated by the upper quartile of the parameter distribution are shown. The cut-off values for each marker and patient numbers are given. The p-values of the Wilcoxon-test are listed. P-values of less than 0.05 are considered to be statistically significant.

relatively poor long-term graft survival (Häyry 1999, Hariharan 2000, Kreis 2001). Chronic inflammation and premature atherosclerosis seem to play a central role in the pathogenesis of complications limiting graft survival (Häyry 1999. Furness 2001, Morris 2001 , Avery 2003).

Numerous studies have shown elevations in inflammatory markers in atherosclerotic disease, including the acute phase reactants SAA and CRP as well as the macrophage product NEOP. These markers seem to correlate with kind and extent of the atherosclerosis (Weis 1994, Erren 1999, Auer 2001, Ridker 2001, Murr 2002, Delanghe 2002). An association between CRP and graft survival could be shown in different settings of solid organ transplantation (Eisenberg 2000. Perez 2000, Labarrere 2002, Sezer 2002, Fink 2002. Teppo 2003). NEOP and SAA seem to be more sensitive and specific markers of acute complications in the carly post-transplant course (Mueller 1999, Grebe 2002. Neumann 2002). However, one early report described the predictive value of NEOP for long-term renal allograft outcome (Reibnegger 1991). In accordance with this study the results from the underlying work confirmed the prognostic significance of $\mathrm{U}$ NEOP for long-term graft survival. In contrast, the acute phase reactants SAA and CRP failed to reach statistical significance.

The reason for the possible higher predictive value of U-NEOP compared to SAA and CRP in our analysis remains unclear. Findings of other groups emphasized the prognostic importance of CRP for renal allograft outcome but did not determine NEOP levels on a regular basis (Perez 2000, Sezer 2002, Fink 2002, Teppo 2003). It is well possible that CRP would reach statistical significance in a larger study population. Differences in the results of the various studies might reflect the heterogeneity of the study populations and immunosuppressive protocols. Our patient group is characterized by a rather high degree in HLA-compatibility but nevertheless a potent immunosuppressive induction therapy. This might reflect increases in the inflammatory burden primarily due to non-rejection related immune responses, in particular antiviral immunity. In these responses macrophage activation and consequently NEOP increases play a key role.

The emerging evidence for the impact of 
macrophage activation on graft survival has been stressed recently and together with previous studies is supported by our findings (Srinivas 2004). However, a consecutive analysis with a higher patient number has been started in order to evaluate the above findings.

In addition, established immunological and nonimmunological risk factors play a major role in the development of chronic allograft nephropathy (Golbabaie 2001, Fellström 2001, Paul 2001). Our data suggest that elevated urinary neopterin levels can identify patients at a higher risk.

\section{Conclusion}

In conclusion the presented results show the impact of the inflammatory burden on graft survival. In particular, elevated post-transplant neopterin values, reflecting activated innate and adaptive immune responses, are associated with a poorer long-term graft outcome.

\section{References}

I Aucr J, Berent R, Labetanig E, Eber B. Serum neopterin and activity of coronary artery disease. Heart Dis 2001; Sept-Oct; 3 (5): 297-301.

2 Avery, RK. Cardiac allograft vasculopathy. N Engl J Med 2003; Aug 18; 349 (9): 829-30.

3 Delanghe JR, Langlois MR, de Bacquer D, Mak R, Capel P, van Renterghem L, de Backer G. Discriminative value of serum amyloid $A$ and other acute-phase proteins for coronary heart disease. Atherosclerosis 2002; Feb 1; 160 (2): 471-6.

4 Eisenberg MS, Chen HJ, Warshofsky MK, Sciacca RR, Wasserman HS, Schwartz A, Rabbani LE. Elevated levels of plasma C-reactive protein are associated with decreased graft survival in cardiac transplant recipients. Circulation 2000; Oct 24; 102 (17): 2100-4.

5 Erren M, Reinecke H, Junker R, Fobker M, Schulte H, Schureck JO, Kropf J, Kerber S, Breithardt G, Assmann, Cullen P. Systemic inflammatory parameters in patients with atherosclerosis of the coronary and peripheral arteries. Arteriosclerosis, Thrombosis and Vascular Biology 1999; 19: 235563.

6 Fellström B. Nonimmune risk factors for chronic renal allograft dysfunction. Transplantation 2001; 71 (11): SS10-16.

7 Fink JC, Onuigbo MA, Blahut SA, Christenson RH, Mann D, Bartlett, ST, Weir MR. Post-transplant serum C-reactive protein and the risk of chronic allograft nephropathy in renal transplant recipients: A pilot case-control study. Am J Kid Dis 2002; May; 39 (5): 1096-101.

8 Furness PN. Histopathology of chronic renal allograft dysfunction. Transplantation 2001; June 15; 71 (11): SS31-36.

9 Golbabaie M, Kayedi M, Najafi I, Ganji MR, Naderi GH, Mehraban D, Hakemi M. Chronic renal allograft dysfunction: Immunologic and nonimmunologic risk factors. Transpl Proc 2001; 33: 2691-2.

10 Grebe SO, Mueller TF. Immune monitoring in organ transplantation using neopterin. Curr Drug Metabol 2002; 3: 189-202.

11 Hariharan S, Johnson CP, Bresnahan BA, Taranto SE, McIntosh MJ, Stablein D. Improved graft survival after renal transplantation in the United States, 1988 to 1996. N Engl J Med 2000 March 2; 342 (9): 605-12.

12 Häyry P, Aavik E, Savolainen H. Mechanisms of chronic rejection. Transpl Proc 1999; 31 (Suppl 7A): $5 \mathrm{~S}-8 \mathrm{~S}$.

13 Kreis HA, Ponticelli C. Causes of late renal allograft loss: chronic allograft dysfunction, death, and other factors. Transplantation 2001 Jun 15; 71 (11 Suppl); SS5-9.

14 Labarrere CA, Lee JB, Nelson DR, Al-Hassani M, Miller SJ, Pitts DE. C-reactive protein, arterial endothelial activation, and development of transplant coronary artery disease: a prospective study. Lancet 2002; Nov 9; 360 (9344): 1462-7.

15 Morris SE. Vascular and cellular mechanisms of chronic renal allograft dysfunction. Transplantation 2001; June 15; 71 (11): SS37-41.

16 Mueller TF. Immunological monitoring following organ transplantation. Papst Science Publishers, Lengerich 1999, 175 pp.

17 Murr C, Widner B, Wirleitner B, Fuchs D. Neopterin as a marker of immune system activation. Curr Drug Metabol 2002; 3: 175-87.

18 Neumann MC, Sprenger H, Grebe SO, Gemsa D, Reibnegger G, Lange $H$, Müller TF. Neopterin, serum amyloid $\mathrm{A}$, and cytokine monitoring after renal transplantation. Pteridines 1998; 9: 113-21.

19 Paul LC. Immunologic risk factors for chronic renal allograft dysfunction. Transplantation 2001: 71 (11): SS17-23.

20 Perez RV, Brown DJ, Katznelson SA, Dubin JA, Müller HG, Chang T, Rudich SM, McVicar JP, Kaysen GA. Pretransplant systemic inflammation and acute rejection after renal transplantation. Transplantation 2000; March 15; 69 (5): 869-74.

21 Reibnegger G, Aichberger C, Fuchs D, Hausen $\Lambda$, Spielberger M, Werner ER, Margreiter R, Wachter 
H. Post-transplant neopterin excretion in renal allograft recipients - a reliable diagnostic aid for acute rejection and a predictive marker of long-term graft survival. Transplantation 1991; 52 (1): 58-63.

22 Ridker PM, Stampfer MJ, Rifai N. Novel risk factors for systemic atherosclerosis: a comparison of C-reactive protein, fibrinogen, homocystein, lipoprotein (a), and standard cholesterol screening as predictors of peripheral arterial disease. JAMA 2001; May 16; 285 (19): 2481-5.

23 Ross R. Atherosclerosis - an inflammatory disease N Engl J Med 1999; Jan 14; 340 (2): 115-126.

24 Sezer S, Ozdemir F, Akcay A, Kulah E, Arat Z, Erdal R, Haberal M. Post-transplant C-reactive protein monitoring predicts chronic rejection and renal allograft survival. Tissue Antigens 2002; Dec: $60(6)$ : 558-9.
25 Srinivas TR, Kubilis PS, Croker BP. Macrophage index predicts short-term renal allograft function and graft survival. Transpl Int 2004; Apr; 17: 195201.

26 Teppo AM, Tornroth T, Honkanen E, GronhagenRiska C. Elevated serum C-reactive protein associates with deterioration of renal function in transplant recipients. Clin Nephrol 2003; Oct; 60 (4): 248-56.

27 Weiss G, Willeit J, Kiechl S, Fuchs D, Jarosch E, Oberhollenzer F, Reibnegger G, Tilz GP, Gerstenbrand F, Wachter H. Increased concentrations of ncoptcrin in carotid atherosclerosis. Atherosclerosis 1991; Apr; 106 (2): 263-71. 